

\title{
Symmetric multistep methods for charged-particle dynamics
}

\author{
ERNST HAIRER ${ }^{1}$ \\ Christian LuBich ${ }^{2}$
}

\author{
${ }^{1}$ Dept. de Mathématiques, Univ. de Genève, CH-1211 Genève 24, Switzerland \\ E-mail address: Ernst.Hairer@unige.ch \\ ${ }^{2}$ Mathematisches Institut, Univ. Tübingen, D-72076 Tübingen, Germany \\ E-mail address: Lubich@na.uni-tuebingen.de.
}

\begin{abstract}
A class of explicit symmetric multistep methods is proposed for integrating the equations of motion of charged particles in an electro-magnetic field. The magnetic forces are built into these methods in a special way that respects the Lagrangian structure of the problem. It is shown that such methods approximately preserve energy and momentum over very long times, proportional to a high power of the inverse stepsize. We explain this behaviour by studying the modified differential equation of the methods and by analysing the remarkably stable propagation of parasitic solution components.
\end{abstract}

Math. classification. 65L06, 65P10, 78A35, 78M25.

Keywords. linear multistep method, charged particle, magnetic field, energy conservation, backward error analysis, modified differential equation, modulated Fourier expansion.

\section{Introduction}

We are interested in the long-term numerical integration of the equations of motion of a charged particle, with position $x(t) \in \mathbb{R}^{3}$ and velocity $v(t)=\dot{x}(t)$ at time $t$, in a magnetic field $B(x)=\nabla \times A(x)$ with the vector potential $A(x) \in \mathbb{R}^{3}$, and under a conservative force $F(x)=-\nabla U(x)$ with the scalar potential $U(x) \in \mathbb{R}$. Assuming physical units where the mass and charge are equal to 1 , the motion is described by the system of second-order differential equations

$$
\ddot{x}=\dot{x} \times B(x)+F(x) .
$$

The energy $E(x, v)=\frac{1}{2}|v|^{2}+U(x)$ is conserved along solutions of (1.1), which follows by taking the inner product with $v=\dot{x}$ on both sides of (1.1) and integrating over time.

The equations (1.1) are the Euler-Lagrange equations $\frac{\mathrm{d}}{\mathrm{d} t} \frac{\partial L}{\partial v}=\frac{\partial L}{\partial x}$ for the Lagrange function $L(x, v)=$ $\frac{1}{2}|v|^{2}+A(x)^{\top} v-U(x)$, as is seen by noting that $v \times B(x)=\left(A^{\prime}(x)^{\top}-A^{\prime}(x)\right) v=A^{\prime}(x)^{\top} v-\frac{\mathrm{d}}{\mathrm{d} t} A(x)$ :

$$
\ddot{x}=A^{\prime}(x)^{\top} \dot{x}-\frac{\mathrm{d}}{\mathrm{d} t} A(x)-\nabla U(x) .
$$

This form of the equations of motion will be the starting point for the construction of the numerical methods of this paper. We note further that with the conjugate momenta $p=\partial L / \partial v=v+A(x)$, one obtains a Hamiltonian system with the non-separable Hamiltonian $H(x, p)=\frac{1}{2}|p-A(x)|^{2}+U(x)$.

A popular numerical method for (1.1) is the Boris method [1], which in its two-step formulation with stepsize $h$ reads

$$
x_{n+1}-2 x_{n}+x_{n-1}=\frac{h}{2}\left(x_{n+1}-x_{n-1}\right) \times B\left(x_{n}\right)-h^{2} \nabla U\left(x_{n}\right) .
$$

This is a second-order method for approximating $x(n h)$ by $x_{n}$. It is essentially an explicit method, since it just requires the solution of a 3-dimensional linear system in each time step. Its energy behaviour has recently been studied in [6], where long-time near-preservation of the total energy is shown for

The research for this article has been partially supported by the Fonds National Suisse, Project No. 200020_159856. 


\section{E.HAIRER \& C. Lubich}

particular cases (constant magnetic field $B$ or quadratic potential $U$ ) but numerical experiments are given that illustrate random walk behaviour or linear growth of the energy error in more general situations.

The Boris method is not a variational integrator unless the magnetic field $B$ is constant [4]. A related variational integrator, which coincides with the Boris method for constant $B$ (or equivalently, affine $A(x)$ ), is constructed like in the interpretation of the Störmer-Verlet method as a variational integrator, by approximating the integral of the Lagrangian $L(x(t), \dot{x}(t))$ over a time step by (a) approximating $x(t)$ as the linear interpolant of the endpoint positions, (b) approximating the integral by the trapezoidal rule, and (c) by extremizing this approximation to the action integral; see, e.g., [7, Chap. VI, Example 6.2] and [11]. This variational integrator reads

$$
x_{n+1}-2 x_{n}+x_{n-1}=\frac{h}{2} A^{\prime}\left(x_{n}\right)^{T}\left(x_{n+1}-x_{n-1}\right)-\frac{h}{2}\left(A\left(x_{n+1}\right)-A\left(x_{n-1}\right)\right)-h^{2} \nabla U\left(x_{n}\right) .
$$

Since a variational integrator can be reformulated as a symplectic integrator for the corresponding Hamiltonian system, the known theory of symplectic integrators shows that this method has very good long-time near-conservation of energy [7]. However, for general $A(x)$, this is an implicit method.

There have been recent efforts to construct explicit symplectic integrators for this problem $[9,10,12]$. While such methods of arbitrary order have been shown to exist, they require many evaluations of $A$ and $A^{\prime}$ or evaluations of higher derivatives of $A$. For example, the fourth-order method proposed in [10] requires 16 evaluations of each $A$ and $A^{\prime}$ per time step. This needs to be put in comparison with the two-stage Gauss-Runge-Kutta method, which is an implicit fourth-order symplectic method. Our objective in this paper is to present and analyse a class of explicit numerical methods that can achieve similarly good energy conservation properties with reduced computational cost.

Our second-order method is a modification of (1.4), where the left-hand side is replaced by a suitable symmetric linear combination of $x_{n+2}, \ldots, x_{n-2}$. This yields an explicit symmetric linear multistep method. To obtain higher-order methods, the simple difference approximations to first- and second-order time derivatives are replaced by symmetric approximations of higher order.

We will show that, under appropriate root conditions for the characteristic polynomial of the coefficients, we obtain in this way a class of explicit numerical integrators with excellent energy and momentum conservation properties. Our results extend those of [5] for symmetric multistep methods for second-order differential equations $\ddot{x}=-\nabla U(x)$; see also [2] for constrained Hamiltonian systems.

We remark that all methods and results of this paper extend without any additional difficulty to systems with a Lagrangian function $L(x, v)=\frac{1}{2} v^{\top} M v+A(x)^{\top} v-U(x)$ for $(x, v) \in \mathbb{R}^{d+d}$ with arbitrary dimension $d \geq 1$, with a positive definite mass matrix $M$. In particular, systems of interacting charged particles are covered, and also gyroscopic mechanical systems.

The paper is organised as follows: Section 2 extends the variational integrator (1.4) in a multistep manner to higher order. The first derivatives of $x$ and $A(x)$ in the vector field are approximated by finite differences. An order barrier, extending the first Dahlquist barrier, is presented for the considered methods. The main results are collected in Section 3. The multistep methods nearly preserve energy and momentum over long intervals of length $\mathcal{O}\left(h^{-p-2}\right)$, where $p$ denotes the order of the method. The construction of methods is explained in Section 4, and numerical experiments illustrating the long-time behaviour of the methods are given in Section 5. The proof of the energy and momentum preservation is split into two parts. Section 6 considers smooth numerical solutions for which a modified differential equation (in the sense of backward error analysis) is derived. A modified energy and a modified momentum are obtained which are formally preserved by the method. The final Section 7 is devoted to bounds on the parasitic solution components of the multistep formula. 


\section{SymmetriC MULTiSTEP METHODS FOR CHARGED-PARTICLE DYNAMICS}

\section{Symmetric multistep methods for charged-particle dynamics}

The variational integrator (1.4) can be interpreted as the Störmer-Verlet method with the first derivatives of $x$ and $A(x)$ approximated by a second order finite difference. This makes the method implicit. To obtain explicit and higher order integrators we extend this method by using multistep techniques.

\subsection{Method formulation}

We consider explicit symmetric multistep methods (with coefficients $\alpha_{j}=\alpha_{-j}, \beta_{j}=\beta_{-j}, \delta_{j}=-\delta_{-j}$ )

$$
\sum_{i=-k-l-1}^{k+l+1} \alpha_{i} x_{n+i}=h^{2} \sum_{i=-l}^{l} \beta_{i}\left(A^{\prime}\left(x_{n+i}\right)^{\top} \frac{1}{h} \sum_{j=-k}^{k} \delta_{j} x_{n+i+j}-\frac{1}{h} \sum_{j=-k}^{k} \delta_{j} A\left(x_{n+i+j}\right)-\nabla U\left(x_{n+i}\right)\right) .
$$

As an approximation to the first derivative we consider the optimal order finite difference formulas with coefficients $\delta_{j}(j=-k, \ldots, k)$ so that we have a derivative approximation of order $2 k$ :

$$
\frac{1}{h} \sum_{j=-k}^{k} \delta_{j} x\left(t_{n}+j h\right)=\dot{x}\left(t_{n}\right)+\mathcal{O}\left(h^{2 k}\right) .
$$

The multistep method (2.1) uses the difference formula also for approximations of the velocity:

$$
v_{n}=\frac{1}{h} \sum_{j=-k}^{k} \delta_{j} x_{n+j}
$$

The $v_{n}$ are computed a posteriori and do not enter the propagation of the numerical solution.

With the multistep formula we associate the generating polynomials

$$
\rho(\zeta)=\zeta^{k+l+1} \sum_{i=-k-l-1}^{k+l+1} \alpha_{i} \zeta^{i}, \quad \sigma(\zeta)=\zeta^{k+l+1} \sum_{i=-l}^{l} \beta_{i} \zeta^{i}
$$

Remark 2.1. Formula (2.1) is based on the linear multistep method

$$
\sum_{i=-k-l-1}^{k+l+1} \alpha_{i} x_{n+i}=h^{2} \sum_{i=-l}^{l} \beta_{i} F_{n+i}
$$

for second order differential equations, where $F_{n}$ is a non standard approximation of the right-hand side of (1.2). Usually, the degree of the polynomial $\sigma(\zeta)$ is at most one less than that of $\rho(\zeta)$. Here it is typically much smaller. The order of method (2.1) is obtained by substituting the exact solution $x\left(t_{n}\right)$ for $x_{n}$, and by examining the defect. The error in the defect due to the finite difference approximation is $\mathcal{O}\left(h^{2 k+2}\right)$. Therefore, the classical order of $(2.5)$ is at most $2 k$. For the limit $h \rightarrow 0$, the method (2.1) tends to a difference equation with characteristic polynomial $\rho(\zeta)$. Zero-stability for $(2.1)$ is therefore the same as that for (2.5).

\subsection{Order barrier}

The particularity of this multistep formula is that there are much fewer coefficients $\beta_{i}$ available than $\alpha_{i}$. An extension of the first Dahlquist barrier (see [3] and [8, Section III.10]) to this situation yields the following result.

Proposition 2.2. The order $p$ of a stable symmetric linear multistep formula with generating polynomials $\rho(\zeta)$ and $\sigma(\zeta)$ of $(2.4)$ is bounded as

$$
p \leq 2 l+2 .
$$




\section{E.HAIRER \& C. LuBICH}

We remark that for order $p=2 k$, we thus need $l \geq k-1$.

Proof. Following the lines of the original proof in [3] we consider the involution $\zeta=(z+1) /(z-1)$ and the transformed polynomials

$$
\begin{aligned}
& R(z)=\left(\frac{z-1}{2}\right)^{2 k+2 l+2} \rho(\zeta)=\sum_{j=0}^{2 k+2 l+2} a_{j} z^{j}, \\
& S(z)=\left(\frac{z-1}{2}\right)^{2 k+2 l+2} \sigma(\zeta)=\sum_{j=0}^{2 k+2 l+2} b_{j} z^{j} .
\end{aligned}
$$

Zero-stability and consistency of the multistep formula imply $a_{2 k+2 l+2}=a_{2 k+2 l+1}=0, a_{2 k+2 l} \neq 0$, and that all coefficients $a_{j}$ have the same sign. In terms of the polynomials $R(z)$ and $S(z)$, order $p$ of the multistep formula is expressed as

$$
\frac{R(z)}{\log ^{2}\left(\frac{z+1}{z-1}\right)}-S(z)=C\left(\frac{2}{z}\right)^{p-2 k-2 l-2}+\mathcal{O}\left(\left(\frac{2}{z}\right)^{p-2 k-2 l-1}\right) \quad \text { for } \quad z \rightarrow \infty .
$$

Since the values $\zeta=0$ and $\zeta=\infty$ are mapped to $z=-1$ and $z=+1$, respectively, the special form of the polynomial $\sigma(\zeta)$ implies that

$$
S(z)=(z+1)^{k+1}(z-1)^{k+1} s(z),
$$

where $s(z)$ is a polynomial of degree $2 l$. We consider the Laurent series of $R(z) /\left(z^{2}-1\right)^{k}$ about $z=\infty$ and we denote a finite part by $\widetilde{R}(z)$ :

$$
\frac{R(z)}{\left(z^{2}-1\right)^{k}}=\sum_{j \leq 2 l} \widetilde{a}_{j} z^{j}, \quad \widetilde{R}(z)=\sum_{j=-2}^{2 l} \widetilde{a}_{j} z^{j} .
$$

Note that $\widetilde{a}_{2 l}=a_{2 k+2 l} \neq 0$. It follows from the positivity of the coefficients of the Neumann series for $1 /\left(1-z^{-2}\right)$ that besides the coefficients of $R(z)$ also those of $\widetilde{R}(z)$ have all the same sign.

Dividing the order relation by $\left(z^{2}-1\right)^{k}$ yields

We also expand

$$
\frac{\widetilde{R}(z)}{\log ^{2}\left(\frac{z+1}{z-1}\right)}-\left(z^{2}-1\right) s(z)=\mathcal{O}\left(\left(\frac{2}{z}\right)^{p-2 l-2}\right) \quad \text { for } \quad z \rightarrow \infty .
$$

$$
\frac{1}{\log ^{2}\left(\frac{z+1}{z-1}\right)}=\frac{z^{2}}{4}-\frac{1}{6}-\frac{3}{180 z^{2}}+\ldots=\frac{z^{2}}{4}-\sum_{m \geq 0} \mu_{m} z^{-m}
$$

and we note that, from Cauchy's formula, we have $\mu_{m} \geq 0$ for all $m \geq 0$.

Let us now assume, by contradiction, that $p>2 l+2$. The principal part of the order relation gives (with $\mu_{-1}=0$ )

$$
\widetilde{R}(z)\left(\frac{z^{2}}{4}-\sum_{m=0}^{2 l} \mu_{m} z^{-m}\right)+\sum_{j=1}^{2 l+2}\left(\sum_{m=j-2}^{2 l} \mu_{m} \widetilde{a}_{m-j}\right) z^{-j}-\left(z^{2}-1\right) s(z)=0 .
$$

Putting $z=1$ yields

$$
\widetilde{R}(1)\left(\frac{1}{4}-\sum_{m=0}^{2 l} \mu_{m}\right)+\sum_{j=1}^{2 l+2}\left(\sum_{m=j-2}^{2 l} \mu_{m} \widetilde{a}_{m-j}\right)=0
$$

This gives a contradiction, because $\widetilde{R}(1) \neq 0$ and the double sum have the same sign, and $\frac{1}{4}-$ $\sum_{m=0}^{2 l} \mu_{m}>0$. 


\section{SymmetriC MULTiSTEP METHODS FOR CHARGED-PARTICLE DYNAMICS}

\section{Almost-conservation of energy and momentum by symmetric multistep methods}

In this section we state our main results, whose proofs will be given in the final two sections of the paper. The following root condition will be essential for the favourable long-time behaviour of methods (2.1).

Definition 3.1. [5] A symmetric multistep method (2.1) with generating polynomials (2.4) is $s$-stable if, apart from the double root at 1 , all zeros of $\rho(\zeta)$ are simple and of modulus one (the letter " $s$ " stands for "simple roots").

The differential equation is equipped with initial values $x(0)=x_{0}$ and $v(0)=v_{0}$. To start the multistep method, starting values $x_{0}, x_{1}, \ldots, x_{2(k+l)+1}$ need to be given. We assume that their errors are $\mathcal{O}\left(h^{p+2}\right)$, as they are when they are computed with a $(p+1)$-th order one-step method:

$$
x_{j}-x(j h)=\mathcal{O}\left(h^{p+2}\right) \quad \text { for } \quad j=0,1, \ldots, 2(k+l)+1 .
$$

Further we assume that the numerical solution values $x_{n}$ stay in a fixed compact subset of the domain on which the vector potential $A(x)$ and the scalar potential $U(x)$ are smooth, and that the velocity approximations $v_{n}$ are bounded by a constant. In view of Theorem 3.2 below, this is for example satisfied if the level sets $\{x: U(x) \leq \mu\}$ are compact and if $A$ and $U$ are smooth on all $\mathbb{R}^{3}$. In the following, the above assumptions are made without further mention.

Theorem 3.2 (Energy conservation). Along numerical solutions obtained by the s-stable symmetric multistep method of order $p$, the total energy $E(x, v)=\frac{1}{2}|v|^{2}+U(x)$ is conserved up to $\mathcal{O}\left(h^{p}\right)$ over times $\mathcal{O}\left(h^{-p-2}\right)$ :

$$
E\left(x_{n}, v_{n}\right)=E\left(x_{0}, v_{0}\right)+\mathcal{O}\left(h^{p}\right) \quad \text { for } \quad n h \leq h^{-p-2} .
$$

The constant symbolized by $\mathcal{O}$ is independent of $n, h$ with $n h \leq h^{-p-2}$.

If the scalar and vector potentials have the invariance properties

$$
U\left(e^{\tau S} x\right)=U(x) \text { and } e^{-\tau S} A\left(e^{\tau S} x\right)=A(x) \quad \text { for all real } \tau
$$

with a skew-symmetric matrix $S$, then the Lagrange function $L(x, v)=\frac{1}{2}|v|^{2}+A(x)^{\top} v-U(x)$ has the invariance $L\left(e^{\tau S} x, e^{\tau S} v\right)=L(x, v)$, and hence Noether's theorem implies that the momentum

$$
M(x, v)=(v+A(x))^{\top} S x
$$

is conserved along solutions of the differential equation (1.2).

Theorem 3.3 (Momentum conservation). Suppose that the system has the invariance (3.2). Along numerical solutions obtained by the s-stable symmetric multistep method of order $p$, the momentum $M(x, v)=(v+A(x))^{T} S x$ is then conserved up to $\mathcal{O}\left(h^{p}\right)$ over times $\mathcal{O}\left(h^{-p-2}\right)$ :

$$
M\left(x_{n}, v_{n}\right)=M\left(x_{0}, v_{0}\right)+\mathcal{O}\left(h^{p}\right) \text { for } n h \leq h^{-p-2} .
$$

The constant symbolized by $\mathcal{O}$ is independent of $n, h$ with $n h \leq h^{-p-2}$.

\section{Construction of higher order methods and implementation}

The construction of symmetric linear multistep methods, suitable for solving charged-particle dynamics, can be done along the following lines:

- We fix $k \geq 1$ and we choose $\left(\delta_{j}\right)_{j=-k}^{k}$ to get an order $2 k$ approximation $(2.2)$ of the first derivative. The coefficients are:

$$
\begin{array}{rlrl}
k=1: & & \frac{1}{2}(-1,0,1) \\
k=2: & \frac{1}{12}(1,-8,0,8,-1)
\end{array}
$$




\section{E.HAIRER \& C. LUBICH}

and for general $k$

$$
\delta_{j}=\frac{(-1)^{j-1}}{j} \frac{k !^{2}}{(k-j) !(k+j) !}, \quad j=1, \ldots, k,
$$

$\delta_{0}=0$, and $\delta_{j}=-\delta_{-j}$ for negative $j$.

- We set $l=k-1$, we arbitrarily choose distinct $a_{j} \in(-1,1)$, and we define

$$
\rho(\zeta)=(\zeta-1)^{2} \prod_{j=1}^{k+l}\left(\zeta^{2}+2 a_{j} \zeta+1\right) .
$$

- We determine the coefficients $\left(\beta_{j}\right)_{j=-l}^{l}$ of $\sigma(\zeta)$ to get order $2 k=2 l+2$.

This procedure gives a method of order $p=2 k$. There is still a freedom in choosing the coefficients $a_{j}$. They can be chosen to make the error constant small and to keep the zeros of $\rho(\zeta)$ well separated.

For the computation of $\sigma(\zeta)$ we note that $\sigma(\zeta)=\zeta^{k+1} \widehat{\sigma}(\zeta)$, where $\widehat{\sigma}(\zeta)$ is a polynomial of degree $2 l$. The order relation for order $p=2 k=2 l+2$, divided by $\zeta^{k+1}$, thus yields (see [8, Theorem III.10.3])

$$
\frac{\rho(\zeta)}{\zeta^{k+1} \log ^{2} \zeta}=\widehat{\sigma}(\zeta)+C_{p+2}(\zeta-1)^{2 l+2}+\mathcal{O}\left((\zeta-1)^{2 l+3}\right) \quad \text { for } \quad \zeta \rightarrow 1 .
$$

Expanding the left-hand side into a Taylor series around $\zeta=1$ gives the polynomial $\widehat{\sigma}(\zeta)$, and at the same time the coefficient $C_{p+2}$ for the error constant $C=C_{p+2} / \sigma(1)=2 C_{p+2} / \rho^{\prime \prime}(1)$.

Method of order 4 . With $k=2$ and $l=1$ we obtain a method of order 4 . Its $\sigma$-polynomial is given by $\sigma(\zeta)=\beta_{0} \zeta^{4}+\beta_{1}\left(\zeta^{5}+\zeta^{3}\right)$ with

$$
\begin{aligned}
& \beta_{0}=\frac{1}{3}\left(20 a_{1} a_{2} a_{3}-4\left(a_{1} a_{2}+a_{1} a_{3}+a_{2} a_{3}\right)-28\left(a_{1}+a_{2}+a_{3}\right)-52\right) \\
& \beta_{1}=\frac{1}{3}\left(2 a_{1} a_{2} a_{3}+14\left(a_{1} a_{2}+a_{1} a_{3}+a_{2} a_{3}\right)+26\left(a_{1}+a_{2}+a_{3}\right)+38\right)
\end{aligned}
$$

and its error constant is

$$
C=\frac{-a_{1} a_{2} a_{3}+9\left(a_{1} a_{2}+a_{1} a_{3}+a_{2} a_{3}\right)+79\left(a_{1}+a_{2}+a_{3}\right)+209}{240\left(1+a_{1}\right)\left(1+a_{2}\right)\left(1+a_{3}\right)} .
$$

By the order barrier of Proposition 2.2 the error constant cannot vanish. Since $C$ is monotonically decreasing in each variable $a_{i} \in(-1,1)$, the error constant is minimal in the limit $a_{1}=a_{2}=a_{3}=1$. To achieve good stability, all roots of $\rho(\zeta)$ should be well separated. We therefore suggest to take the values

$$
a_{1}=-0.7, \quad a_{2}=0.1, \quad a_{3}=0.9
$$

for a method of order 4 .

The implementation of the symmetric multistep methods must be done carefully. A straightforward implementation of the formula (2.1) gives non-satisfactory behaviour caused by round-off errors. It is recommended to introduce variables $v_{j}$ and $a_{j}$ according to

$$
x_{j+1}=x_{j}+h v_{j}, \quad v_{j+1}=v_{j}+h a_{j},
$$

and to write the left-hand sum in (2.1) in terms of the variables $a_{j}$. The sum $\sum_{j=-k}^{k} \delta_{j} x_{n+j}$ can be written in terms of $v_{j}$. 


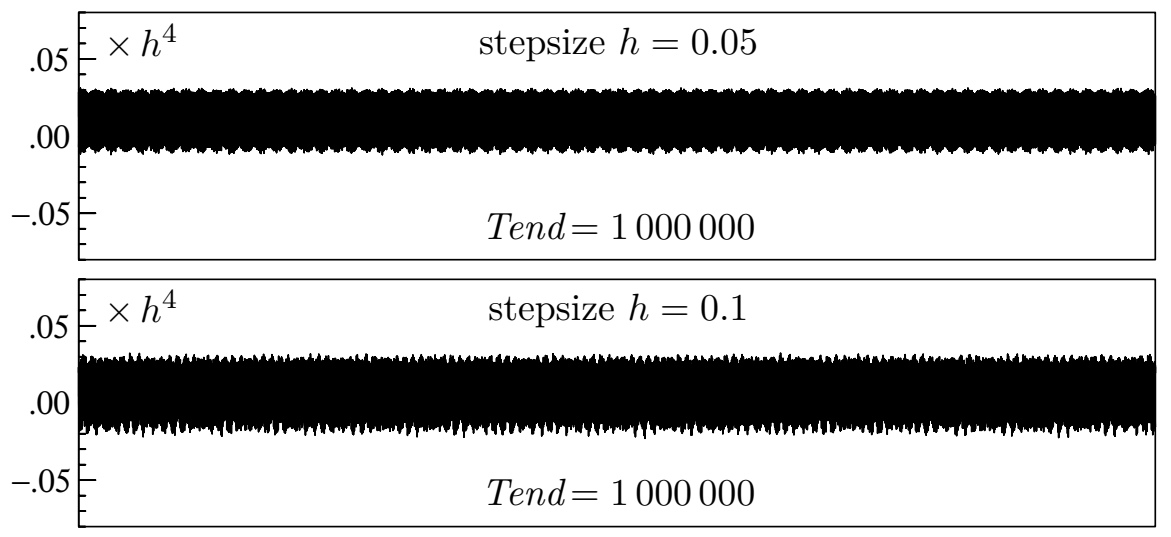

FiguRE 5.1. Error in the energy as a function of time for two different time stepsizes.

\section{Numerical experiments}

A common test problem is the $2 \mathrm{D}$ dynamics of a charged particle in a static electromagnetic field, where the potential $U(x)$ and the field $B(x)$ are given by

$$
U(x)=\frac{1}{100 \sqrt{x_{1}^{2}+x_{2}^{2}}}, \quad B(x)=\left(0,0, \sqrt{x_{1}^{2}+x_{2}^{2}}\right)^{\top} .
$$

We have $B(x)=\nabla \times A(x)$ with

$$
A(x)=\frac{1}{3}\left(-x_{2} \sqrt{x_{1}^{2}+x_{2}^{2}}, x_{1} \sqrt{x_{1}^{2}+x_{2}^{2}}, 0\right)^{\top} .
$$

As initial values we take

$$
x(0)=(0.0,1.0,0.1)^{\top}, \quad v(0)=(0.09,0.05,0.20)^{\top} .
$$

To this problem we apply the symmetric multistep method of order 4 constructed in Section 4 . To illustrate that the error in the energy is bounded by $\mathcal{O}\left(h^{4}\right)$ we plot in Figure 5.1 the error, scaled by $h^{4}$, for two different stepsizes $h=0.1$ and $h=0.05$. The computation is done over the long time interval $\left[0,10^{6}\right]$ and no drift in the energy can be observed.

Besides the energy we also consider the momentum

$$
M(x, v)=\left(v_{1}+A_{1}(x)\right) x_{2}-\left(v_{2}+A_{2}(x)\right) x_{1} .
$$

Its error is plotted in Figure 5.2. Again we observe a $\mathcal{O}\left(h^{4}\right)$ behaviour and an excellent long-time conservation of the numerical momentum.

\section{Backward error analysis for smooth numerical solutions}

Throughout this section we consider linear multistep methods (2.1) of order $p=2 k$. We extend Section 3 of [5] to the problem of this paper and study the exceptional case of numerical solutions $\left(x_{n}\right)$ for which

$$
x_{n}=y(n h)+\mathcal{O}\left(h^{N}\right) \quad \text { for a smooth function } y(t),
$$

where $N \gg p$ and smoothness is understood to mean that all derivatives of $y(t)$ are bounded independently of $h$. (Strictly speaking, this refers to families of functions $y(t)$ parametrized by $h$.) The situation (6.1) is met only for very special starting values, whereas general numerical solutions contain oscillatory terms which correspond to powers of the roots of $\rho(\zeta)$ other than 1 . Nevertheless, the 


\section{E.HAIRER \& C. Lubich}

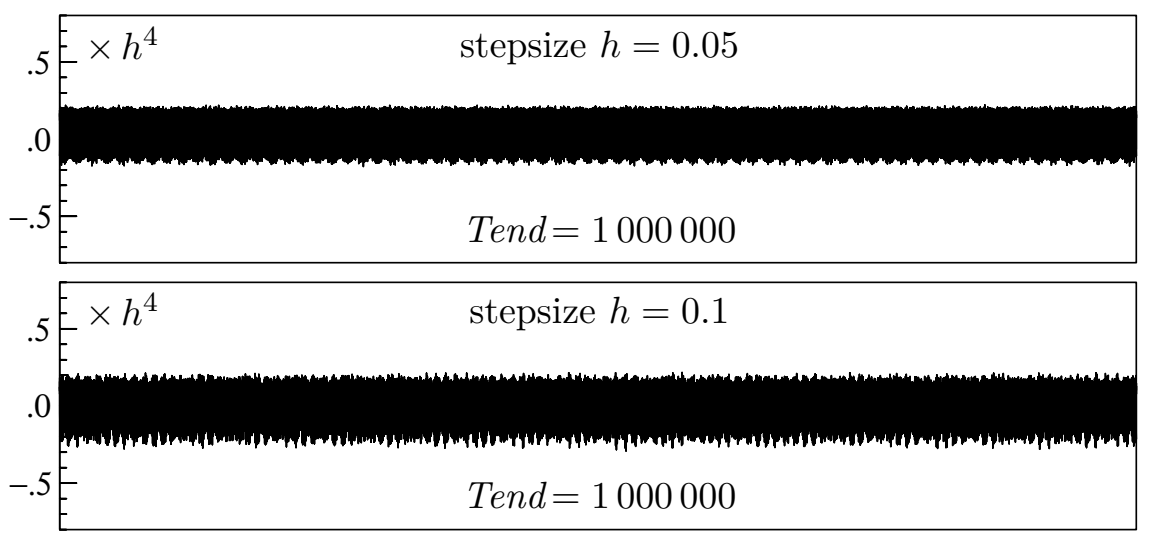

FiguRE 5.2. Error in the momentum as a function of time for two different time stepsizes.

idealized situation of no parasitic terms gives already much insight into the conservation properties of the method, in a technically simpler framework than the general case.

\subsection{Modified differential equation}

The following result is proved in the same way as Theorem 4 of [5].

Proposition 6.1. There exist unique $h$-independent functions $f_{j}(x, v)$ such that, for every truncation index $N$, every solution of

$$
\ddot{y}=\dot{y} \times B(y)-\nabla U(y)+h^{p} f_{p}(y, \dot{y})+\ldots+h^{N-1} f_{N-1}(y, \dot{y})
$$

satisfies

$$
\begin{aligned}
\sum_{i=-k-l-1}^{k+l+1} \alpha_{i} y(t+i h) & =\sum_{i=-l}^{l} \beta_{i}\left(h A^{\prime}(y(t+i h))^{T} \sum_{j=-k}^{k} \delta_{j} y(t+(i+j) h)\right. \\
& \left.-h \sum_{j=-k}^{k} \delta_{j} A(y(t+(i+j) h))-h^{2} \nabla U(y(t+i h))\right)+\mathcal{O}\left(h^{N+2}\right) .
\end{aligned}
$$

Moreover, $f_{j}(x, v)=0$ for all odd $j$.

With the standard convergence theory of linear multistep methods [8], this implies that (6.1) holds on intervals of length $\mathcal{O}(1)$ if it is satisfied for the starting values.

\subsection{Modified energy}

We now show that the modified differential equation (6.2) has a formal first integral close to the total energy $E(x, v)$.

Proposition 6.2. There exists a formal modified energy

$$
E_{h}(x, v)=E(x, v)+h^{p} E_{p}(x, v)+h^{p+2} E_{p+2}(x, v)+\ldots
$$

such that its truncation at the $\mathcal{O}\left(h^{N}\right)$ term satisfies

$$
\frac{\mathrm{d}}{\mathrm{d} t} E_{h}(y(t), \dot{y}(t))=\mathcal{O}\left(h^{N}\right)
$$

along solutions of the modified differential equation (6.2). 


\section{SymmetriC MULTiSTEP METHODS FOR CHARGED-PARTICLE DYNAMICS}

We remark that Propositions 6.1 and 6.2 imply, for smooth numerical solutions (6.1) and their $p$ th order velocity approximations (2.3),

$$
E\left(x_{n}, v_{n}\right)=E\left(x_{0}, v_{0}\right)+\mathcal{O}\left(h^{p}\right)+\mathcal{O}\left(t h^{N}\right) .
$$

Proof. The proof extends the proof of Proposition 1 in [5]. With $D=\mathrm{d} / \mathrm{d} t$ and the shift operator $e^{h D}$, with $\delta(\zeta)=\sum_{j=-k}^{k} \delta_{j} \zeta^{j}$, and with the expansion $\rho\left(e^{x}\right) /\left(x^{2} \sigma\left(e^{x}\right)\right)=\left(1+\gamma_{p} x^{p}+\gamma_{p+2} x^{p+2}+\ldots\right)$, we write equation $(6.3)$ as

$$
\begin{aligned}
& \left(1+\gamma_{p} h^{p} D^{p}+\gamma_{p+2} h^{p+2} D^{p+2}+\ldots\right) \ddot{y}= \\
& A^{\prime}(y)^{\top} h^{-1} \delta\left(e^{h D}\right) y-h^{-1} \delta\left(e^{h D}\right) A(y)-\nabla U(y)+\mathcal{O}\left(h^{N}\right),
\end{aligned}
$$

where the left-hand side contains only even-order derivatives of $y$ by the symmetry of the method. We multiply both sides of (6.6) with $\dot{y}^{\top}$, so that on the right-hand side we have

$$
\dot{y}^{\top} h^{-1}\left(A^{\prime}(y)^{\top} \delta\left(e^{h D}\right) y-\delta\left(e^{h D}\right) A(y)\right)-\frac{\mathrm{d}}{\mathrm{d} t} U(y)+\mathcal{O}\left(h^{N}\right) .
$$

The left-hand side is the time derivative of an expression in which the appearing second and higher derivatives of $y$ can be substituted as functions of $(y, \dot{y})$ via the modified differential equation (6.2). So far, this is the argument in [5].

It remains to show that the first term of (6.7) can also be written as the time derivative of a function of $(y, \dot{y})$, up to $\mathcal{O}\left(h^{N}\right)$. We note that for the Euclidean inner product $\langle\cdot, \cdot\rangle$ we have

$$
\left\langle\dot{y}, A^{\prime}(y)^{\top} \delta\left(e^{h D}\right) y\right\rangle=\left\langle A^{\prime}(y) \dot{y}, \delta\left(e^{h D}\right) y\right\rangle=\left\langle D A(y), \delta\left(e^{h D}\right) y\right\rangle,
$$

which removes the transpose in (6.7). For a function $f$ that is analytic at 0 , partial integration shows that for time-dependent smooth functions $u$ and $v$,

$$
\langle f(h D) u, v\rangle-\langle u, f(-h D) v\rangle \text { is a total derivative up to } \mathcal{O}\left(h^{N}\right) \text { for arbitrary } N
$$

of a function that depends on $u, v$ and their higher time derivatives. With $f(z)=z^{-1} \delta\left(\mathrm{e}^{z}\right), u=D A(y)$, and $v=D y$ this yields that

$$
\left\langle\delta\left(e^{h D}\right) A(y), D y\right\rangle-\left\langle D A(y), \delta\left(e^{h D}\right) y\right\rangle
$$

is a total derivative up to an $O\left(h^{N}\right)$ error (using the skew-symmetry of the coefficients $\delta_{j}$, so that $f(-z)=f(z))$. Together with (6.8) this shows that the first term of (6.7) is a total derivative (up to $\left.\mathcal{O}\left(h^{N}\right)\right)$ of a function of $y, \dot{y}$ and of higher derivatives of $y$ that can be substituted as functions of $(y, \dot{y})$ via the modified differential equation (6.2). Moreover, this function is of size $\mathcal{O}\left(h^{p}\right)$, because $h^{-1} \delta\left(e^{h D}\right) y=\dot{y}+\mathcal{O}\left(h^{p}\right)$.

Collecting the terms that appear when (6.6) is scalarly multiplied with $\dot{y}$, we thus obtain (6.5) with a function of the form (6.4).

Remark 6.3. For the Boris method, one obtains instead

$$
\frac{\mathrm{d}}{\mathrm{d} t} E_{h}(y(t), \dot{y}(t))=h^{2} \dot{y}(t)^{\top}(\dddot{y}(t) \times B(y(t)))+\ldots,
$$

where the term on the right-hand side is generally not a time derivative of a function of $(y, \dot{y})$.

\subsection{Modified momentum}

We further obtain that the modified differential equation (6.2) has a formal first integral close to the momentum $M(x, v)$ of $(3.3)$. Note that in general this is not a quadratic first integral.

Proposition 6.4. Under the invariance condition (3.2), there exists a formal modified momentum

$$
M_{h}(x, v)=M(x, v)+h^{p} M_{p}(x, v)+h^{p+2} M_{p+2}(x, v)+\ldots
$$




\section{E.HAirer \& C. Lubich}

such that its truncation at the $\mathcal{O}\left(h^{N}\right)$ term satisfies

$$
\frac{\mathrm{d}}{\mathrm{d} t} M_{h}(y(t), \dot{y}(t))=\mathcal{O}\left(h^{N}\right)
$$

along solutions of the modified differential equation (6.2).

Proof. The proof is analogous to the previous proof when (6.6) is multiplied with $(S y)^{\top}$ instead of $\dot{y}^{\top}$, using that $y^{\top} S \nabla U(y)=0$ and $A^{\prime}(y) S y=S A(y)$ by $(3.2)$.

\section{Long-term analysis of parasitic solution components}

We now consider general numerical solutions obtained by the symmetric multistep method. We adapt the programme of [5] and [2] (following more closely the simplified version of [2]) to derive the modified equations for the principal and the parasitic solution components and to use their structure to derive long-term bounds for the parasitic solution components. In the proofs we concentrate on the aspects related to the magnetic field, which is not present in [5] and [2]. With the bounds for the parasitic components, the almost-conservation of energy and momentum over long times can then be concluded from Propositions 6.2 and 6.4.

\subsection{Principal and parasitic modified equations}

Let $\zeta_{0}=1$ be the double root of the characteristic polynomial $\rho(\zeta)$ and $\zeta_{ \pm 1}, \ldots, \zeta_{ \pm(k+l)}$ the simple roots on the unit circle, ordered such that $\zeta_{-i}=\bar{\zeta}_{i}$. We denote by $\mathcal{I}$ the set of integers $i$ with $0<|i| \leq k+l$. The following result is proved in the same way as Theorem 5 in [5] and Proposition 4 in [2].

Proposition 7.1 (Modified equations). Consider an s-stable symmetric linear multistep method (2.1) of order $p$. Then, there exist $h$-independent matrix-valued functions $F_{i, j}(y, v)$, such that for every truncation index $M$ and for every solution of the combined system (6.2) and

$$
\dot{z}_{i}=\left(h F_{i, 1}(y, \dot{y})+\cdots+h^{M-1} F_{i, M-1}(y, \dot{y})\right) z_{i}, \quad i \in \mathcal{I},
$$

with initial values satisfying $z_{-i}(0)=\bar{z}_{i}(0)$, the following holds: as long as $\left\|z_{i}(t)\right\| \leq \vartheta$ for all $i \in \mathcal{I}$ (with sufficiently small $\vartheta$ ), the function ${ }^{1}$

$$
\widehat{x}(t)=y(t)+\sum_{i \in \mathcal{I}} \zeta_{i}^{t / h} z_{i}(t)
$$

satisfies

$$
\begin{aligned}
\sum_{i=-k-l-1}^{k+l+1} \alpha_{i} \widehat{x}(t+i h) & =h^{2} \sum_{i=-l}^{l} \beta_{i}\left(A^{\prime}(\widehat{x}(t+i h))^{\top} \frac{1}{h} \sum_{j=-k}^{k} \delta_{j} \widehat{x}(t+(i+j) h)\right. \\
- & \left.\frac{1}{h} \sum_{j=-k}^{k} \delta_{j} A(\widehat{x}(t+(i+j) h))-\nabla U(\widehat{x}(t+i h))\right)+\mathcal{O}\left(h^{N+2}+h^{M+1} \vartheta+h \vartheta^{2}\right) .
\end{aligned}
$$

\footnotetext{
${ }^{1}$ The analogous expression in [5] has a sum over an index set that includes also finite products of $\zeta_{i}$. This is not necessary for the investigations of the present work. We here follow the simplified approach of [2].
} 


\section{SymmetriC MULTiSTEP METHODS FOR CHARGED-PARTICLE DYNAMICS}

We do not include the proof, which is very similar to that of the corresponding results in $[2,5]$, but we note for later use that the functions $y$ and $z_{i}$ satisfy the modulation equations

$$
\begin{aligned}
h^{-2} \frac{\rho}{\sigma}\left(e^{h D}\right) y & =A^{\prime}(y)^{\top} h^{-1} \delta\left(e^{h D}\right) y-h^{-1} \delta\left(e^{h D}\right) A(y)-\nabla U(y)+\mathcal{O}\left(h^{N}\right) \\
h^{-1} \frac{\rho}{\sigma}\left(\zeta_{i} e^{h D}\right) z_{i} & =\left.\frac{\mathrm{d}}{\mathrm{d} \varepsilon}\right|_{\varepsilon=0} A^{\prime}\left(y+\varepsilon z_{i}\right)^{\top} \delta\left(e^{h D}\right) y \\
& +A^{\prime}(y)^{\top} \delta\left(\zeta_{i} e^{h D}\right) z_{i}-\delta\left(\zeta_{i} e^{h D}\right)\left(A^{\prime}(y) z_{i}\right)-h \nabla^{2} U(y) z_{i}+\mathcal{O}\left(h^{M} \vartheta\right), \quad i \in \mathcal{I} .
\end{aligned}
$$

As in Lemma 1 of [5] we obtain the following.

Lemma 7.2 (Initial values). Consider an s-stable symmetric multistep method (2.1). To every set of starting values $x_{0}, \ldots, x_{2(k+l)+1}$ satisfying $x_{j}-x(j h)=\mathcal{O}\left(h^{s}\right)(j=0, \ldots, 2(k+l)+1)$ with $1 \leq s \leq p+2$ there exist (locally) unique initial values $y(0), h \dot{y}(0), z_{i}(0)(i \in \mathcal{I})$ for the system (6.2) and (7.1) such that its solution exactly satisfies

$$
x_{j}=y(j h)+\sum_{i \in \mathcal{I}} \zeta_{i}^{j} z_{i}(j h) \quad \text { for } j=0, \ldots, 2(k+l)+1 .
$$

These initial values satisfy $z_{-i}(0)=\overline{z_{i}}(0)$ and

$$
y(0)-x(0)=\mathcal{O}\left(h^{s}\right), \quad h \dot{y}(0)-h \dot{x}(0)=\mathcal{O}\left(h^{s}\right), \quad z_{i}(0)=\mathcal{O}\left(h^{s}\right) .
$$

With initial values and functions $y(t), z_{i}(t)$ as in Lemma 7.2 the numerical solution after one step satisfies, for $j=2(k+l)+2$,

$$
x_{j}=y(j h)+\sum_{i \in \mathcal{I}} \zeta_{i}^{j} z_{i}(j h)+\mathcal{O}\left(h^{N+2}+h^{M+1} \vartheta+h \vartheta^{2}\right) .
$$

This is an immediate consequence of Proposition 7.1.

\subsection{Bounds on parasitic solution components}

We set out to prove that the parasitic solution components $z_{i}(t)$ remain bounded and small on long time intervals.

Proposition 7.3 (Almost-invariants). Under the assumptions of Proposition 7.1, there exist h-independent matrix-valued functions $E_{i, l}(y, v)$ such that for every truncation index $M$ and for every solution of the combined system (6.2) and (7.1) the functions

$$
K_{i}\left(y, v, z_{i}\right)=\left\|z_{i}\right\|^{2}+\bar{z}_{i}^{\top}\left(h^{2} E_{i, 2}(y, v)+\ldots+h^{M-1} E_{i, M-1}(y, v)\right) z_{i}
$$

for $i \in \mathcal{I}$ are almost-invariants; more precisely, we have

$$
K_{i}\left(y(t), \dot{y}(t), z_{i}(t)\right)=K_{i}\left(y(0), \dot{y}(0), z_{i}(0)\right)+\mathcal{O}\left(t h^{M} \vartheta^{2}\right), \quad i \in \mathcal{I}
$$

as long as $(y(t), \dot{y}(t))$ stays in a compact set and $\left\|z_{i}(t)\right\| \leq \vartheta$ for $i \in \mathcal{I}$.

Proof. We multiply (7.4) with $z_{i}^{*}=\bar{z}_{i}^{\top}$ and take the imaginary part. We first consider the terms on the right-hand side. Since the Hessian $\nabla^{2} U(y)$ is real symmetric, $z_{i}^{*} \nabla^{2} U(y) z_{i}$ is real, so that its imaginary part vanishes.

With $w=h^{-1} \delta\left(e^{h D}\right) y$ and $V_{w}(y)=-A(y)^{\top} w$, we have

so that also

$$
\left.\frac{\mathrm{d}}{\mathrm{d} \varepsilon}\right|_{\varepsilon=0} A^{\prime}\left(y+\varepsilon z_{i}\right)^{\top} \delta\left(e^{h D}\right) y=-h \nabla^{2} V_{w}(y) z_{i}
$$

$$
\left.\Im z_{i}^{*} \frac{\mathrm{d}}{\mathrm{d} \varepsilon}\right|_{\varepsilon=0} A^{\prime}\left(y+\varepsilon z_{i}\right)^{\top} \delta\left(e^{h D}\right) y=0
$$




\section{E.HAIRER \& C. Lubich}

Next we show that $\Im z_{i}^{*} A^{\prime}(y)^{\top} \delta\left(\zeta_{i} e^{h D}\right) z_{i}-\Im z_{i}^{*} \delta\left(\zeta_{i} e^{h D}\right)\left(A^{\prime}(y) z_{i}\right)$ is a total derivative (up to $\mathcal{O}\left(h^{L}\right)$ for arbitrary $L$ ). We expand

$$
\delta\left(\zeta_{i} e^{-\mathrm{i} x}\right)=\mathrm{i} \sum_{j=0}^{\infty} c_{i, j} x^{j}
$$

with real coefficients $c_{i, j}$, because by the anti-symmetry of the coefficients $\delta_{j}$ and $\left|\zeta_{i}\right|=1$ we have $\delta\left(\zeta_{i} e^{-\mathrm{i} x}\right)=-\delta\left(\bar{\zeta}_{i} e^{\mathrm{i} x}\right)=-\overline{\delta\left(\zeta_{i} e^{-\mathrm{i} x}\right)}$. We then have

$$
\Im z_{i}^{*} \delta\left(\zeta_{i} e^{h D}\right)\left(A^{\prime}(y) z_{i}\right)=\Im z_{i}^{*} \mathrm{i} \sum_{j=0}^{\infty} c_{i, j}(\mathrm{i} h D)^{j}\left(A^{\prime}(y) z_{i}\right),
$$

which (by partial integration) differs from

$$
\Im \mathrm{i} \sum_{j=0}^{\infty} c_{i, j}\left((-\mathrm{i} h D)^{j} z_{i}^{*}\right) A^{\prime}(y) z_{i}=\Im z_{i}^{*} A^{\prime}(y)^{\top} \mathrm{i} \sum_{j=0}^{\infty} c_{i, j}(\mathrm{i} h D)^{j} z_{i}=\Im z_{i}^{*} A^{\prime}(y)^{\top} \delta\left(\zeta_{i} e^{h D}\right) z_{i} .
$$

only by a total derivative of a linear combination of inner products of $z_{i}$ and $A^{\prime}(y) z_{i}$ and their derivatives. Moreover, the term for $j=0$ cancels.

Concerning the left-hand side, in the proof of Proposition 7 in [2] it is shown by a similar argument that $\Im z_{i}^{*} h^{-1} \frac{\rho}{\sigma}\left(\zeta_{i} e^{h D}\right) z_{i}$ can be written as the total derivative of a sum of a constant nonzero multiple of $\left\|z_{i}\right\|^{2}$ and of a quadratic function of $z_{i}$ and its derivatives multiplied with a positive power of $h$.

Expressing the higher derivatives of $y$ and $z_{i}$ as function of $(y, \dot{y})$ and $z_{i}$ via the modified differential equations (6.2) and (7.1), we therefore obtain functions $K_{i}$ of the stated form such that $\frac{d}{d t} K_{i}\left(y(t), \dot{y}(t), z_{i}(t)\right)=\mathcal{O}\left(h^{M} \vartheta^{2}\right)$.

We collect the assumptions that are required for proving the boundedness of the parasitic solution components.

(A1): The multistep method (2.1) is symmetric and of order $p=2 k$. All roots of $\rho(\zeta)$, with the exception of the double root $\zeta_{0}=1$, are simple and of modulus one.

(A2): The scalar potential $U$ and the vector potential $A$ are defined and smooth in an open neighbourhood of a compact set $K$.

(A3): The starting approximations $x_{0}, \ldots, x_{2(k+l)+1}$ are such that the initial values for the system (6.2), (7.1) obtained from Lemma 7.2 satisfy

$$
y(0) \in K, \quad\|\dot{y}(0)\| \leq M, \quad\left\|z_{i}(0)\right\| \leq \vartheta / 2, \quad i \in \mathcal{I} .
$$

(A4): The numerical solution $\left\{x_{n}\right\}$, for $0 \leq n h \leq T$, stays in a compact set $K_{0}$ that has a positive distance to the boundary of $K$. $[2]$.

From Proposition 7.3 we then obtain the following result with the same proof as for Theorem 3 in

Theorem 7.4 (Long-time bounds for the parasitic components). Assume (A1)-(A4). For sufficiently small $h$ and $\vartheta$ and for fixed truncation indices $N$ and $M$ that are large enough such that $h^{N}=\mathcal{O}\left(\vartheta^{2}\right)$ and $h^{M-1}=\mathcal{O}(\vartheta)$, there exist functions $y(t)$ and $z_{i}(t)$ for $i \in \mathcal{I}$ on an interval of length

$$
T=\mathcal{O}\left(\vartheta^{-1}\right)
$$

such that

- $x_{n}=y(n h)+\sum_{i \in \mathcal{I}} \zeta_{i}^{n} z_{i}(n h)$ for $0 \leq n h \leq T$; 


\section{SymmetriC MULTiSTEP METHODS FOR CHARGED-PARTICLE DYNAMICS}

- on every subinterval $[n h,(n+1) h)$ the functions $y(t)$ and $z_{i}(t)$ for $i \in \mathcal{I}$ are a solution of the system (6.2), (7.1);

- the functions $y(t), h \dot{y}(t)$ and $z_{i}(t)$ for $i \in \mathcal{I}$ have jump discontinuities of size $\mathcal{O}\left(h \vartheta^{2}\right)$ at the grid points nh;

- for $0 \leq t \leq T$, the parasitic components are bounded by

$$
\left\|z_{i}(t)\right\| \leq \vartheta, i \in \mathcal{I} \text {. }
$$

Proof. The proof is a simplified variant of the proof of Theorem 3 in [2]. To define the functions $y(t)$ and $z_{i}(t)$ on the interval $[n h,(n+1) h)$ we consider the consecutive numerical solution values $x_{n}, x_{n+1}, \ldots, x_{n+k-1}$. We compute initial values for the system (6.2), (7.1) according to Proposition 7.2, and we let $y(t), z_{i}(t)$ be its solution on $[n h,(n+1) h)$. By $(7.7)$ this construction yields jump discontinuities in $y(t), h \dot{y}(t)$ and $z_{i}(t)$ of size $\mathcal{O}\left(h \vartheta^{2}\right)$ at the grid points.

It follows from Proposition 7.3 that the functions $K_{i}\left(y(t), \dot{y}(t), z_{i}(t)\right)$ remain constant up to an error of size $\mathcal{O}\left(h^{M+1} \vartheta^{2}\right)$ on the interval $[n h,(n+1) h)$. The jump discontinuity in $z_{i}(t)$ of size $\mathcal{O}\left(h \vartheta^{2}\right)$ at the grid points induces a jump discontinuity of size $\mathcal{O}\left(h \vartheta^{3}\right)$ in $K_{i}\left(y(t), \dot{y}(t), z_{i}(t)\right)$. Adding the error terms $\mathcal{O}\left(h^{M+1} \vartheta^{2}\right)$ and $\mathcal{O}\left(h \vartheta^{3}\right)$ over $n$ subintervals, we find that with $t=n h$

$$
K_{i}\left(y(t), \dot{y}(t), z_{i}(t)\right) \leq K_{i}\left(y(0), \dot{y}(0), z_{i}(0)\right)+C_{1} t h^{M} \vartheta^{2}+C_{2} t \vartheta^{3}
$$

as long as $\left\|z_{i}(t)\right\| \leq \vartheta$ for $i \in \mathcal{I}$. Noting that there is a factor $h^{2}$ in the terms of $K_{i}\left(y, v, z_{i}\right)$ that depend on $(y, v)$, this implies together with Proposition 7.3

$$
\left\|z_{i}(t)\right\|^{2} \leq\left\|z_{i}(0)\right\|^{2}+C_{1} t h^{M} \vartheta^{2}+C_{2} t \vartheta^{3}+C_{3} h^{2} \vartheta^{2} .
$$

The assumption $\left\|z_{i}(t)\right\| \leq \vartheta$ for $i \in \mathcal{I}$ is certainly satisfied as long as $C_{1} t h^{M}+C_{2} t \vartheta \leq 1 / 4$ and $C_{3} h^{2} \leq 1 / 4$, so that the right-hand side of the above estimates is bounded by $\vartheta^{2}$. This proves the estimate for $\left\|z_{i}(t)\right\|$ for $t=\mathcal{O}\left(\vartheta^{-1}\right)$, and at the same time it guarantees recursively that the above construction of the functions $y(t)$ and $z_{i}(t)$ is feasible over such times.

\subsection{Proof of the main results}

Theorems 3.2 and 3.3 follow from Theorem 7.4 and Propositions 6.2 and 6.4 on using the short argument of Section 7.4 of [2], which we include for the convenience of the reader.

For the piecewise smooth function $y(t)$ of Theorem 7.4 we have

$$
E_{h}(y(t), \dot{y}(t))=E_{h}(y(0), \dot{y}(0))+\mathcal{O}\left(t h^{N}\right)+\mathcal{O}\left(t h^{-1} \vartheta^{2}\right),
$$

where the first error term results from Proposition 6.2 and the second error term results from the discontinuity at the grid points, which is of size $\mathcal{O}\left(\vartheta^{2}\right)$ for the derivative $\dot{y}(t)$ by Theorem 7.4. Since we have $n$ crossings of discontinuities until $t=n h$, this error accumulates to $\mathcal{O}\left(n \vartheta^{2}\right)=\mathcal{O}\left(t h^{-1} \vartheta^{2}\right)$. By the bounds for the parasitic components $z_{i}$ we have

$$
x_{n}=y(n h)+\mathcal{O}(\vartheta) \quad \text { and } \quad v_{n}=\dot{y}(n h)+\mathcal{O}\left(h^{-1} \vartheta+h^{p}\right)
$$

because the differentiation formula is of order $p$. We therefore obtain

$$
E_{h}\left(x_{n}, v_{n}\right)=E_{h}\left(x_{0}, v_{0}\right)+\mathcal{O}\left(t h^{N}\right)+\mathcal{O}\left(t h^{-1} \vartheta^{2}\right)+\mathcal{O}\left(h^{-1} \vartheta+h^{p}\right) .
$$

If the errors in the starting values are $\mathcal{O}\left(h^{p+2}\right)$, then assumption (A3) is satisfied with $\vartheta=\mathcal{O}\left(h^{p+2}\right)$ by Lemma 7.2, and hence $T=\mathcal{O}\left(h^{-p-2}\right)$ in Theorem 7.4. Finally, Theorem 3.2 now follows by using the $\mathcal{O}\left(h^{p}\right)$ estimate between the modified energy $E_{h}$ and the original energy $E$ as given by Proposition 6.2.

Theorem 3.3 is obtained in the same way using Proposition 6.4. 


\section{E.HAIRER \& C. Lubich}

Acknowledgement. We thank our colleague Martin Gander for drawing our attention to the longtime behaviour of the Boris algorithm.

\section{References}

[1] J. P. Boris. Relativistic plasma simulation-optimization of a hybrid code. Proceeding of Fourth Conference on Numerical Simulations of Plasmas, pages 3-67, November 1970.

[2] P. Console, E. Hairer, and C. Lubich. Symmetric multistep methods for constrained Hamiltonian systems. Numerische Mathematik, 124:517-539, 2013.

[3] G. Dahlquist. Stability and error bounds in the numerical integration of ordinary differential equations. Trans. of the Royal Inst. of Techn., Stockholm, Sweden, 130, 1959.

[4] C. L. Ellison, J. W. Burby, and H. Qin. Comment on "Symplectic integration of magnetic systems": A proof that the Boris algorithm is not variational. J. Comput. Phys., 301:489-493, 2015.

[5] E. Hairer and C. Lubich. Symmetric multistep methods over long times. Numer. Math., 97:699-723, 2004.

[6] E. Hairer and C. Lubich. Energy behaviour of the Boris method for charged-particle dynamics. Submitted for publication, 2017.

[7] E. Hairer, C. Lubich, and G. Wanner. Geometric Numerical Integration. Structure-Preserving Algorithms for Ordinary Differential Equations. Springer Series in Computational Mathematics 31. Springer-Verlag, Berlin, 2nd edition, 2006.

[8] E. Hairer, S. P. Nørsett, and G. Wanner. Solving Ordinary Differential Equations I. Nonstiff Problems. Springer Series in Computational Mathematics 8. Springer, Berlin, 2nd edition, 1993.

[9] Y. He, Z. Zhou, Y. Sun, J. Liu, and H. Qin. Explicit $K$-symplectic algorithms for charged particle dynamics. Phys. Lett. A, 381(6):568-573, 2017.

[10] M. Tao. Explicit high-order symplectic integrators for charged particles in general electromagnetic fields. J. Comput. Phys., 327:245-251, 2016.

[11] S. D. Webb. Symplectic integration of magnetic systems. J. Comput. Phys., 270:570-576, 2014.

[12] R. Zhang, H. Qin, Y. Tang, J. Liu, Y. He, and J. Xiao. Explicit symplectic algorithms based on generating functions for charged particle dynamics. Physical Review E, 94(1):013205, 2016. 